\title{
Primary Intracranial Malignant Melanoma with Extracranial Metastasis
}

\author{
Kengo Hirota, M.D., Chika Yoshimura, M.D., Osami Kubo, M.D., Hidetoshi Kasuya, M.D. \\ Department of Neurosurgery, Tokyo Women's Medical University Medical Center East, Tokyo, Japan
}

We report a case of primary intracranial malignant melanoma (PIMM) with extracranial metastases. The patient was an 82-yearold woman diagnosed with PIMM under the left cerebellar tentorium. We performed a tumor resection followed by gamma knife surgery. An magnetic resonance imaging at 11 months after surgery showed a local intracranial recurrence. At 12 months, vertebral metastasis was suspected, and 2-[fluorine-18]-fluoro-2-deoxy-D-glucose positron emission tomography/computed tomography (FDG-PET/CT) showed multiple extracranial metastases. She died at 13 months after surgery. Although extracranial metastases of PIMM are extremely rare, we should carefully follow up extracranial metastases together with intracranial ones, especially by FDG$\mathrm{PET} / \mathrm{CT}$, even at an early asymptomatic stage.

Key Words : Intracranial malignant melanoma · Extracranial metastasis · Positron emission tomography.

\section{INTRODUCTION}

Primary intracranial malignant melanoma (PIMM) is a rare tumor of the central nervous system $(\mathrm{CNS})^{2}$. Metastatic intracranial malignant melanoma (MIMM) from cutaneous malignant melanoma (CMM) is a frequent occurrence. PIMM is difficult to diagnose because PIMM and MIMM are similar in radiological and pathological appearance ${ }^{6,15)}$. On the other hand, extracranial metastasis of PIMM is extremely rare. Both timing and sites of recurrent PIMM are unpredictable. Here, we report a case of PIMM with extracraninal metastasis.

\section{CASE REPORT}

\section{History and examination}

An 82-year-old women came to the department because magnetic resonance imaging (MRI), followed up every one year for asymptomatic cerebral infarction, showed mass lesion under the left cerebellar tentorium (Fig. 1). One month later, on her admission, she had cerebellar symptoms such as nausea and gait disturbance, and the mass lesion showed as rapidly enlarged on the MRI. Past medical history was unremarkable. There was no mucocutaneous or ocular pigmented lesion on the skin. No mass lesion was shown one year before admission. The mass lesion with niveau formation and a

• Received: June 3, 2015 •Revised: August 8, 2015 •Accepted: September 23, 2015

- Address for reprints : Kengo Hirota, M.D.

Department of Neurosurgery, Tokyo Women's Medical University Medical Center East, 2-1-10 Nishiogu, Arakawa-ku, Tokyo 116-8567, Japan Tel : +81-3-3810-1111, Fax : +81-3-3819-8680, E-mail : k2bumps@gmail.com

This is an Open Access article distributed under the terms of the Creative Commons Attribution Non-Commercial License (http://creativecommons.org/licenses/by-nc/4.0) which permits unrestricted non-commercial use, distribution, and reproduction in any medium, provided the original work is properly cited. 

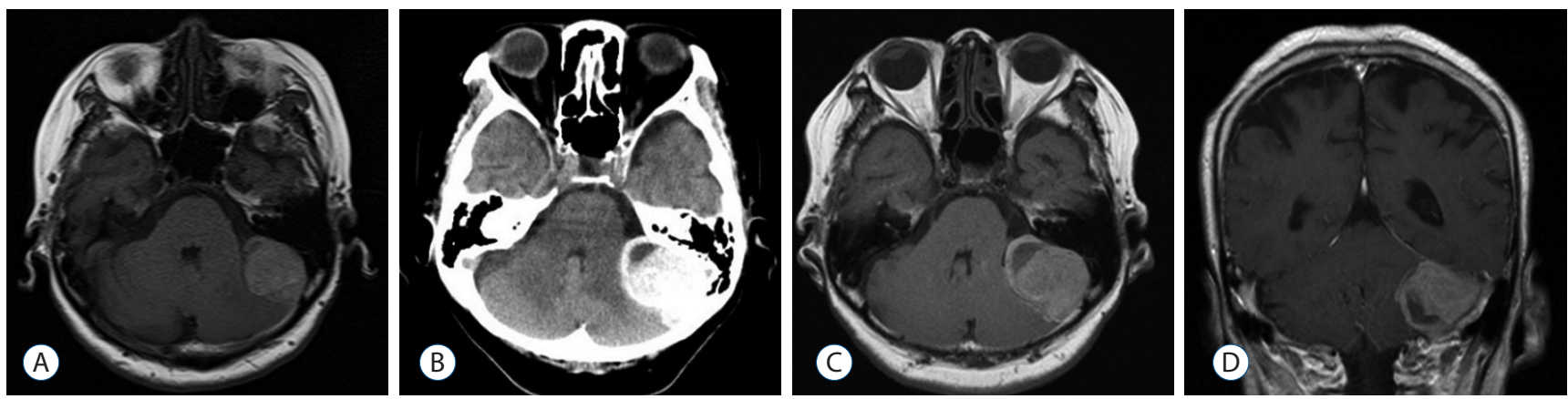

Fig. 1. Axial T1-weighted MRI (A) shows an extra-axial mass lesion under the left cerebellar tentorium one month before admission. Axial CT (B), T1weighted MRI (C) and Coronal Gd-enhanced T1-weighted MRI (D) on admission show the enlarged mass lesion with niveau formation at one month later. MRI : magnetic resonance imaging, CT : computed tomography.

maximum diameter of $5 \mathrm{~cm}$ showed high density on computed tomography (CT), a slightly high intensity on T1 MRI and a low intensity on T2 MRI. It did not show enhancement after gadolinium administration (Fig. 1). 2-[fluorine-18]-fluoro-2-deoxy-D-glucose positron emission tomography/computed tomography (FDG-PET/CT), chest and abdominal enhanced CT did not detect any malignant lesion such as an extracranial malignant melanoma (Fig. 2).

\section{Surgery and pathological examination}

We performed a near total resection, but some residual tumor attached to the sigmoid sinus and the petrosal vein remained. A black solid and encapsulated nodular tumor was found under the left cerebellar tentorium and appeared to originate from the pia mater of the cerebellum (Fig. 3). The tumor tissue was stained with H\&E and other immunohistochemical stains. The aggregates of melanin were contained in the cytoplasm. H\&E after decolorization of melanin showed atypical cells, necrosis and abnormal mitosis. Immunohistochemical examinations were positive for HMB-45, Melan-A and S-100 protein. The MIB-1 (Ki-67) staining index was approximately $8 \%$ (Fig. 3). The final pathological diagnosis was PIMM.

\section{Postoperative course}

She became ambulatory and was discharged. We performed gamma knife surgery at a total dose of 21 Gy for the residual tumor of the sigmoid sinus and the petrosal vein. An MRI at 11 months after surgery showed local recurrence at

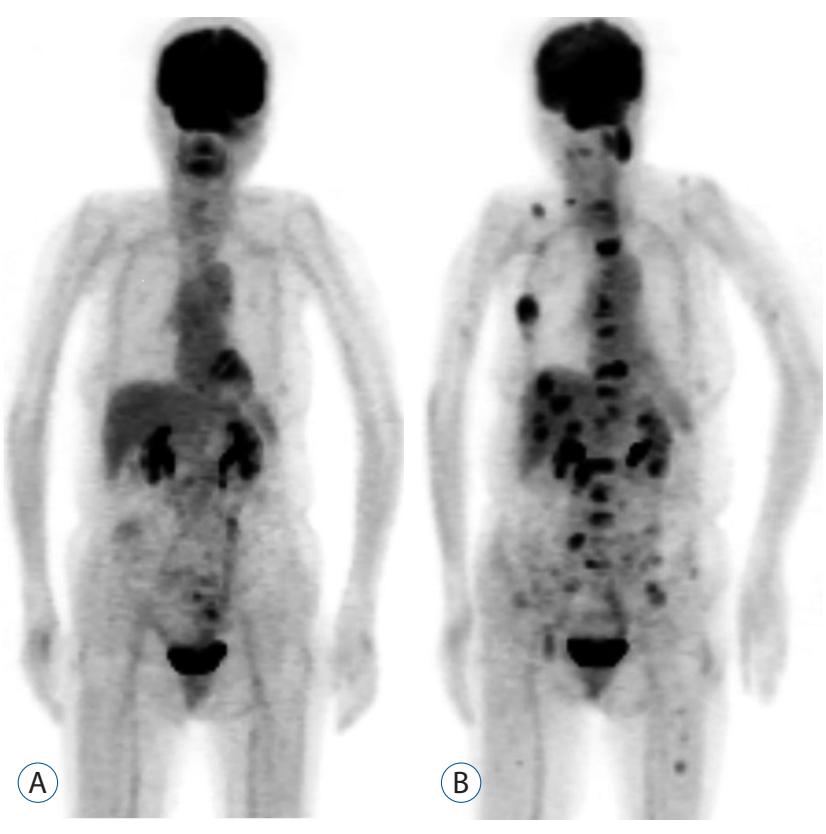

Fig. 2. Whole body FDG-PET/CT (A) on admission shows no malignant lesions such as extracranial malignant melanoma. FDG-PET/CT (B) at 12 months after the surgery shows FDG uptake in multiple metastatic lesions. FDG-PET/CT : 2-[fluorine-18]-fluoro-2-deoxy-D-glucose positron emission tomography/computed tomography.

the anterolateral edge of the left cerebellar tentorium. We performed gamma knife surgery at a total dose of 24 Gy for the recurrent tumor. At 12 months, she experienced back pain, and vertebral metastasis was suspected after MRI. Therefore, FDG-PET/CT, chest and abdominal CT for a whole body examination was performed and showed multiple metastases in the left neck, the right latissimus dorsi muscle, the right collarbone, the liver, multiple vertebral bodies and the left thigh bone (Fig. 2). Recurrence in the brain and brainstem was difficult to distinguish in the FDG- 

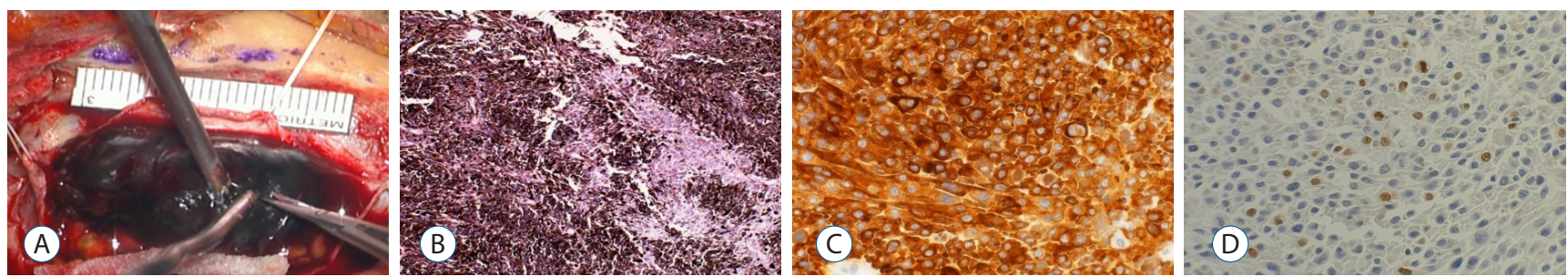

Fig. 3. Intraoperative image (A) shows the black colored tumor on the left cerebellar tentorium. Photomicrographs. The tumor cells arranged in fascicles intersperse with melanin-containing cells $(B, H \& E, \times 200)$. The tumor cells are positive for Melan-A (C, $\times 400)$. The MIB-1 (Ki-67) staining index is approximately $8 \%(D, \times 400)$.

PET/CT. Her general condition continued to be good, and we performed an open biopsy for diagnosis of the tumor lesion in the right latissimus dorsi muscle. Pathological examination of the intramuscular tumor was the same as those previously described. The MIB-1 staining index was approximately $7 \%$. At 13 months after surgery, the patient died from cryptogenic cardiopulmonary arrest.

\section{DISCUSSION}

PIMM accounts for approximately $0.1 \%$ of all intracranial tumors ${ }^{2}$. PIMM predominantly originates from the leptomeningeal melanocytes. These cells are found underneath the brain and the brainstem, inside the ventricles, at the optic chiasm, in the grooves of the various lobes, in the substantia nigra and in the choroid ${ }^{16}$. Primary spinal cord melanoma is rare, but has been reported previously ${ }^{9)}$.

It is not easy to differentiate PIMM from MIMM because they overlap in radiological and pathological appearance ${ }^{15)}$. It has been reported that the following factors are proposed for establishing a diagnosis of PIMM: (i) no malignant melanoma outside the CNS; (ii) involvement of the leptomeninges; (iii) intramedullary spinal lesions; (iv) hydrocephalus; (v) tumor in the pituitary or pineal gland; and (vi) a single intracerebral lesion ${ }^{6}$. PIMM is generally a diagnosis of exclusion. In our case, there were no skin or eye melanoma, neither the FDG-PET/CT or chest and abdominal enhanced CT detected malignant lesions in the other parts of her body. It has been reported that MIMM often shows extensive necrosis, more obvious cytologic malignancy, a higher mitotic index and a higher MIB-1 staining index. An MIB-1 staining index may provide useful information to help classification. The MIB-1 staining index of MIMM (range, 17-38 per $10 \mathrm{HPFs}$ ) was higher than that of PIMM (range, 2-15 per $10 \mathrm{HPFs})^{1)}$. In our case, the MIB-1 staining index was approximately $8 \%$. Thus, PIMM was more likely. It has also been reported that PIMM have a better prognosis than MIMM. The median survival for patients with MIMM is only 5-6 months while patients with PIMM survive 9-24 months. Individuals have been reported to survive for more than 5 years ${ }^{5,6,12-14,16)}$.

Many cases with PIMM die from recurrence of intracranial lesions. Extracranial metastasis of PIMM is extremely rare ${ }^{3)}$. It has reported that of 168 cases with extracranial metastasis in adults 68 were cases of glioblastoma, 49 of meningeal tumors, 22 of medulloblastoma, and other cases were of ependymoma, pituitary gland, oligodendroglioma, neuroblastoma, germinoma and hemangioblastoma ${ }^{7}$. The lungs, pleura, lymph nodes, bone, and liver are the most common sites of extracranial metastases of glioblastoma ${ }^{4,11}$.

The routes of extracranial metastasis are not clear. Risk factors for extracranial metastasis include previous craniotomy, ventricular systemic shunting, high-grade tumor histology, radiation therapy, long interval since the primary therapy and tumoral relapse ${ }^{17)}$. In our case, the patient received craniotomy and some residual tumor attached to the sigmoid sinus and the petrosal vein remained. It is quite possible that the residual tumor invading the venous sinus and the dural sinus was the cause of the hematogenous dissemination.

CMM can spread to any organ. The most common sites of clinical presentation are the skin, subcutaneous tissue and lymph nodes followed by lung, liver and bone. It is quite possible that CT, MRI, FDG-PET/CT and skeletal scintigraphy provide clinically useful information to detect the metastasis 
of CMM. Especially, a whole body FDG-PET/CT is a non-invasive, high-resolution molecular imaging technique that can detect CMM metastasis based on abnormal cellular glucose uptake $^{10)}$. However, FDG-PET/CT has limitations with small (less than $1 \mathrm{~cm}$ ), pulmonary and brain metastasis ${ }^{3)}$. On the other hand, the optimal time point for detecting metastatic malignant melanoma is not certain. It has been reported that the optimal timing to perform FDG-PET/CT in high risk $\mathrm{CMM}$ is at 6 months after surgery because all recurrences at an early asymptomatic stage were found between 7-14 months after surgery ${ }^{8)}$. In the case of PIMM, a head MRI is routinely performed to check for recurrent PIMM. Therefore, we concluded that whole body FDG-PET/CT should also be performed to screen for extracranial PIMM metastasis even at an early asymptomatic stage after surgery.

\section{CONCLUSION}

We reported a case of PIMM with extracranial metastasis. Although extracranial PIMM metastasis is extremely rare, we should carefully follow up extracranial metastases together with intracranial ones, especially with FDG-PET/CT, even at an early asymptomatic stage.

\section{References}

1. Brat DJ, Giannini C, Scheithauer BW, Burger PC : Primary melanocytic neoplasms of the central nervous systems. Am J Surg Pathol 23 : 745-754, 1999

2. Brat DJ, Perry A : Melanocytic lesions, Louis DN, Ohgaki H, Wiestler OD, Cavenee WK (ed) : WHO classification of tumours of the central nervous system, 4th ed. Lyon: IARC, 2007, pp181-183

3. Dinesh SM, Suneetha $B$, Sen $A$ : A rare case of primary malignant mela- noma of clivus with extensive skeletal metastasis demonstrated on 18FFDG PET/CT. Indian J Nucl Med 28 : 234-236, 2013

4. Glasauer FE, Yuan RH : Intracranial tumors with extracranial metastases. Case report and review of the literature. J Neurosurg $20: 474-493$, 1963

5. Greco Crasto $S$, Soffietti R, Bradac GB, Boldorini R : Primitive cerebral melanoma: case report and review of the literature. Surg Neurol 55 : 163-168; discussion 168, 2001

6. Hayward RD : Malignant melanoma and the central nervous system: a guide for classification based on clinical findings. J Neurol Neurosurg Psychiatr 39 : 526-530, 1976

7. Hoffman HJ, Duffner PK : Extraneural metastases of central nervous system tumors. Cancer 56(7 Suppl) : 1778-1782, 1985

8. Koskivuo IO, Seppänen MP, Suominen EA, Minn HR : Whole body positron emission tomography in follow-up of high risk melanoma. Acta Oncol 46 : 685-690, 2007

9. Kim MS, Yoon DH, Shin DA : Primary spinal cord melanoma. J Korean Neurosurg Soc 48 : 157-161, 2010

10. Larcos G, Maisey MN : FDG-PET screening for cerebral metastases in patients with suspected malignancy. Nucl Med Commun 17 : 197198, 1996

11. Lun M, Lok E, Gautam S, Wu E, Wong ET : The natural history of extracranial metastasis from glioblastoma multiforme. J Neurooncol 105 : 261-273, 2011

12. Nakagawa H, Hayakawa T, Niiyama K, Nii Y, Yoshimine T, Mori S : Longterm survival after removal of primary intracranial malignant melanoma. Case report. Acta Neurochir (Wien) $101: 84-88,1989$

13. Rodriguez y Baena R, Gaetani P, Danova M, Bosi F, Zappoli F : Primary solitary intracranial melanoma: case report and review of literature. Surg Neurol 38 : 26-37, 1992

14. Shah I, Imran M, Akram R, Rafat S, Zia K, Emaduddin M : Primary intracranial malignant melanoma. J Coll Physicians Surg Pak 23 : 157159, 2013

15. Wadasadawala T, Trivedi S, Gupta T, Epari S, Jalali R : The diagnostic dilemma of primary central nervous system melanoma. J Clin Neurosci 17 : 1014-1017, 2010

16. Wang J, Guo ZZ, Wang YJ, Zhang SG, Xing DG : Microsurgery for the treatment of primary malignant intracranial melanoma: a surgical series and literature review. Eur J Surg Oncol 40 : 1062-1071, 2013

17. Yasuhara T, Tamiya T, Meguro T, Ichikawa T, Sato Y, Date I, et al. : Glioblastoma with metastasis to the spleen--case report. Neurol Med Chir (Tokyo) 43 : 452-456, 2003 